\title{
Association Between Iron Deficiency and Febrile Seizure? A Case Control Study in Tehran, Iran
}

Samileh Noorbakhsh ( $\square$ samileh_noorbakhsh@yahoo.com )

Pediatric Infectious Disease Depart, Iran University of Medical Sciences

Research

Keywords: febrile convulsion, children, Ferritin, Iron deficiency

Posted Date: January 11th, 2021

DOl: https://doi.org/10.21203/rs.3.rs-142050/v1

License: (c) (i) This work is licensed under a Creative Commons Attribution 4.0 International License. Read Full License 


\section{Abstract}

Objective: Febrile convulsion (FC) is the most common form of seizures disorder (3-4\%) in childhood with good prognosis. Some evidences indicate that iron deficiency (ID) might play a role in initiation of FC. The aim of this case-control study was to evaluate, the role for ID in pathogenesis of FC (febrile convulsion). We studied $35 \mathrm{FC}$ children and 35 controls (febrile diseases without convulsion)

Methods; In this case-control study, 70 chidren admitted in pediatrics department (2014-2016) were studied. Cases included 35 children with FC ( mean age: $2.191 \pm 0946$ years); $62.9 \%$ male,37.1\% female .The Controls consisted 35 children with febrile diseases without convulsion (( mean age: $1.93 \pm 1.433$ years) $65.7 \%$ male,34.3\% female.

Two militter of peripheral blood collected within the first day of admission in hospital. The blood were tested for $\mathrm{CBC}, \mathrm{HB}, \mathrm{MCV}, \mathrm{MCH}, \mathrm{MCHC}$ in 2 groups. The remaining blood in an acid-propylene tube were centrifuged and serum was preserved in- $80^{\circ} \mathrm{C}$.Serum ferritin was estimated by EIAS test for the cases and controls .Data were analyzed by SPSS 13.HB „MCVMCH,MCHC, and serum ferritin level were compared between 2 groups using appropriate statistical tools . The Ferritin level cut-off in serum for differentiating between 2 groups was constructed by receiver-operating-characteristic curve(ROC). Sensitivity, specificity, PPV, NPV, of test was calculated.

Results: The mean age of cases were $2.191 \pm 0946$ vs $1.93 \pm 1.433$ years in controls .Mean Hemoglobin level (11.6 \pm 079 vs $11.86 \pm 071 ; p$ value $=0.2)$; $\mathrm{MCV}(75.8 \pm 4.3$ vs $77.62 \pm 4.1, p$ value $=0.08)$; serum ferritin levels ( $54.57 \pm 24$ vs $58.31 \pm 23$, p value $=0.64$ ) had not significant difference between 2 groups. The ferritin level $36 \mathrm{ng} / \mathrm{ml}$; had $74.3 \%$ sensitivity, 20\% specifity, 56\% PPV, 52\% NPV, Positive likelihood Ratio: 1.3; Negative likelihood Ratio: 0.93 to discriminate 2 groups. In our opinion, ID could not lead to FC in all children, but in some cases with genetic basis; ID raises the threshold for seizures. Due to high prevalence of ID (26\%) especially in your Iranian population, adding the Iron to diet might be helpful in decreasing FC in susceptible cases.

Conclusion: The present study is the first to report the ferritin cut off level which discriminates the FC cases from non convulsive febrile children. Ferritin cut off $(36 \mathrm{ng} / \mathrm{ml}$ has an acceptable sensitivity $(74.3 \%$ ) but poor specifity (20\%) and just 56\% PPV, and 52\% NPV to differentiate the FC cases from non convulsive febrile children. In our opinion, ID could not lead to FC in all children, but in some cases with genetic basis; ID raises the threshold for seizures. Due to high prevalence of ID (26\%) especially in your Iranian population, adding the Iron to diet, might be helpful in decreasing FC in susceptible cases.

\section{Introduction}

One of the most common childhood convulsive disorders are FC (Febrile convulsions); With wide incidence rate in world; $2-4 \%$ in United states; $9-10 \%$ in and Japan, the highest rate; $14 \%$ in Guam. (1-3) FC is a seizure associated with a febrile illness in the absence of central nervous system infections or acute electrolyte abnormalities in 6-60 months old children without previous afebrile seizures $(2$, 
3).Multiple etiologic factors are considered for FC (4-9) ID reduces the metabolism of some neurotransmitters.On the other hand, fever may aggravate negative effects of ID on the brain (11) .Confilcting results are seen from previous studies about relationship between ID and FC (11-13)

Fc with incidence rate $2-5 \%$ is the most common seizures in Iranian children between 5 months -6 years old (14-17) In spite of the efforts of ministry of health and medical education of Iran for supplemental iron for all Iranian infants at least for 1-2 years ; and also for girls, the prevalence of ID is considerable (14-15) In a recent systematic search study by Akbari et al (14) )in population-based studies estimates the prevalence rate $26.9 \%$ for ID in Iran ,with $14 \%$ prevalence rate in young population (< 18 years) including $7.9 \%$ in males and $8.5 \%$ among females ( $<18$ yeas). Contradictory results between ID and FC have shown in Iran like the other parts of world. (16-21) First, Mahyar et al (16) reported the risk factors for FC in Iranian children. Recently, Heydarian et al explained the First FC and Clinical risk factors in our country .Veisani et al discussed the familial history and recurrence of FC in a Systematic Review and Meta-Analysis(17) Some studies by Iranian authors tried to evaluate role of ID in FC children (18-25) Some authors reported the ID were more frequent in children with FC in compare with non convulsive febrile and normal children $(18,22,23,25)$. On the contrary, Salehi Talebian, Amirsalari shown that ID plays no role in pediatric FC. (19-21). We carried out this prospective case-control study to report the ferritin cut off level which could discriminate the FC cases from non convulsive febrile children.

\section{Materials And Methods}

This prospective case/control study was conducted during 2 years (2014-2016) in pediatric wards in Rasoul, 3th level referral hospital in Tehran, Iran

This study was approved by the by the scientific advisory and ethical committees of IranUniversity of Medical Sciences. A written informed consent forms were signed by the parents, and the procedures involved complied with Declaration of Helsinki study.before including the children in the study.

Seventhy febrile children were selected from cases were admitted in pediatric ward during 2 years .A check list was completed for the subjects who included complete history such as age, sex, seizure history, seizure type, systemic diseases. One militter of blood in 2 groups in the first admission were tested for $\mathrm{CBC}$, The serum ferritin for the cases and controls were studied by ELISA (enzyme-linked immunosorbent assay) in first day of admission. These values enrolled in the questionnaire.

\section{Cases Definition:}

Inclusion criteria: Thirty five patients with final diagnosis of febrile convulsion (without underlying disease and normal CSF analysis) selected as cases.

All febrile seizure group had the first bout of single generalized febrile seizure prolonged $<15$ minutes..

Exclusion criteria: All children with known causes for convulsion after complete clinical studies (e.g. electrolyte imbalance, leukemia, metabolic disorders, chronic diseases, malnutrition, );CNS infection 
(meningitis, encephalitis,brain abscess) ) excluded from study. Also we excluded all Children who had mental retardation, brain anomalies, brain tumor ,atypical convulsion, focal seizure, chronic diseases, moderate to severe malnutrition,

\section{Controls}

Thirty five febrile children without convulsion and normal CNS examination (unconsciousness $\mathrm{r} / \mathrm{no}$ meiningeal signs and symptoms / no other abnormal neurologic deficits),negative bacterial culture (CSF/Blood) .Viral infection was the final diagnosis febrile control group.

\section{Lab test:}

Two militter of peripheral blood collected within the first day of admission in hospital.The blood were tested for $\mathrm{CBC}, \mathrm{HB}, \mathrm{MCV}, \mathrm{MCH}, \mathrm{MCHC}$ in2 groups. The remaining blood in an acid-propylene tube were centrifuged and serum was preserved in- $80^{\circ} \mathrm{C}$.Serum ferritin was estimated by EIAS test for the cases and controls

\section{Statistical Analysis}

All analyses were conducted using SPSS, version 13.5.Quantitative variables were summarized as mean \pm standard deviation (SD) and qualitative variables were counted and expressed as percentages. The Student's t test was used to determine significant differences in meansof all continuous variables.. Chisquare test was performed to compare proportion between 2 or more discrete variables. $P<0.05$ was considered statistically significant.

ROC (receiver-operating-characteristic curve) illustrated and the cut-off level for serum Ferritin, Sensitivity, specificity, PPV, NPV, of test was calculated.

\section{Results}

Of 70 admitted children, the cases included 35 children (mean age: $2.191 \pm 0946$ years); $62.9 \%$ male,37.1\% female who were referred due to FC .Also, 35 children who were hospitalized due to febrile diseases without convulsion enrolled in this trial as controls (mean age: $1.93 \pm 1.433$ years) $65.7 \%$ male, $34.3 \%$ female.

No significant difference observed in age and gender between FC cases and controls ( $p$ value $=0.3$; 08).No significant difference were determined between 2 groups for mean the Hemoglobin level $(11.6 \pm$ 079 vs $11.86 \pm 071 ; p$ value $=0.2) ; \mathrm{MCV}(75.8 \pm 4.3$ vs $77.62 \pm 4.1$, $\mathrm{p}$ value $=0.08)$; and $\mathrm{MCH}(26.2 \pm 1.5$ vs $27.3 \pm 2.8, \mathrm{p}$ value $=0.07)$

The mean level for serum ferritin in 2 groups was $56.4 \mathrm{ng} / \mathrm{ml}$. All data Compared between cases and controls in Table- 1.

Table -1: Comparison the data between cases and controls 


\begin{tabular}{|lllllll|}
\hline Data & $\mathrm{Hb}$ & \multicolumn{3}{c}{$\mathrm{MCV} / \mathrm{MCH}$} & \multicolumn{2}{c|}{ Ferritin level $(\mathrm{ng} / \mathrm{ml})$} \\
\cline { 2 - 7 } & Case I & Control & Case & Control & Casel & Control ferritin \\
\hline Mean & 11.6 & $11.86 \pm$ & $75.8 / 26.2$ & $77.62 / 27.3$ & 54.57 & 58.31 \\
\hline Standard deviation & 079 & 071 & $4.3 / 1.5$ & $4.1 / 2.1$ & 24 & 23 \\
\hline P value & 0.2 & & 0.08 & & 0.64 & \\
\hline
\end{tabular}

Figure-1:show the area under curve (AUC) .AUC was $0.442(0.306-0.578, \mathrm{P}$ value $=0.4)$. According to this curve, the calculated cut-off level for ferritin was $36 \mathrm{ng} / \mathrm{ml}$.; it had $74.3 \%$ sensitivity, $20 \%$ specifity, $56 \%$ PPV, 52\% NPV. The Positive likelihood Ratio: 1.3; Negative likelihood Ratio: 0.93 was calculated.

Area Under the Curve

Test Result Variable(s): FERRITIN

\begin{tabular}{|lllll|}
\hline Area & Std. Error ${ }^{\mathrm{a}}$ & Asymptotic Sig. & \multicolumn{3}{l|}{ Asymptotic 95\% Confidence Interval } \\
\cline { 3 - 5 } & & & Lower Bound & Upper Bound \\
\hline .442 & .069 & .404 & .306 & .578 \\
\hline
\end{tabular}

The test result variable(s): FERRITIN has at least one tie between the positive actual state group and the negative actual state group. Statistics may be biased.

a. Under the nonparametric assumption

b. Null hypothesis: true area $=0.5$

Coordinates of the Curve

Test Result Variable(s): FERRITIN 


\begin{tabular}{|c|c|c|}
\hline Positive if Greater Than or Equal To ${ }^{a}$ & Sensitivity & 1 - Specificity \\
\hline 9.00 & 1.000 & 1.000 \\
\hline 15.00 & .971 & .971 \\
\hline 20.50 & .943 & .971 \\
\hline 21.50 & .914 & .971 \\
\hline 23.00 & .886 & .971 \\
\hline 26.00 & .886 & .943 \\
\hline 29.00 & .886 & .914 \\
\hline 32.50 & .829 & .886 \\
\hline 36.00 & .743 & .800 \\
\hline 37.50 & .686 & .771 \\
\hline 39.00 & .657 & .771 \\
\hline 41.50 & .543 & .714 \\
\hline 43.50 & .514 & .714 \\
\hline 44.50 & .514 & 657 \\
\hline 46.00 & .457 & .600 \\
\hline 47.50 & .457 & .571 \\
\hline 48.50 & .429 & .514 \\
\hline 49.50 & .429 & .486 \\
\hline 52.00 & .400 & .371 \\
\hline 54.50 & .343 & .343 \\
\hline 56.50 & .314 & .343 \\
\hline 59.00 & .286 & .343 \\
\hline 62.00 & .257 & .314 \\
\hline 64.50 & .257 & .286 \\
\hline 66.50 & .229 & .286 \\
\hline 69.00 & .229 & .257 \\
\hline 71.50 & .200 & .200 \\
\hline 74.00 & .171 & .200 \\
\hline
\end{tabular}




\begin{tabular}{|lll|}
77.50 & .114 & .143 \\
82.50 & .086 & .143 \\
89.00 & .086 & .114 \\
109.50 & .086 & .086 \\
128.00 & .086 & .057 \\
135.00 & .057 & .057 \\
145.00 & .057 & .029 \\
160.00 & .029 & .029 \\
173.50 & .000 & .029 \\
\hline 178.00 & .000 & .000 \\
\hline
\end{tabular}

The test result variable(s): FERRITIN has at least one tie between the positive actual state group and the negative actual state group.

a. The smallest cutoff value is the minimum observed test value minus 1 , and the largest cutoff value is the maximum observed test value plus 1 . All the other cutoff values are the averages of two consecutive ordered observed test values

The Cut off for ferritin ( $<36 \mathrm{ng} / \mathrm{ml}$ ) observed in $30 \%$ (9/35) of convulsive cases in compare with $21 \%$ $(7 / 35)$ I n controls, without significant difference $(54.57 \pm 24$ vs $58.31 \pm 23$, p value $=0.64):$ Table-2

Table-2: The ferritin Cut off $(<36 \mathrm{ng} / \mathrm{ml})$ compared between cases and controls

\begin{tabular}{|lll|}
\hline & \multicolumn{2}{l|}{ Ferritin level $(<36 \mathrm{ng} / \mathrm{ml})$} \\
\hline Febrile convulsion & Positive & Negative \\
\hline Positive & 9 & 26 \\
\hline Negative & 7 & 28 \\
\hline
\end{tabular}

\section{Discussion}

The present study is the first to report the ferritin cut off level which discriminates the FC cases from nonconvulsive febrile children. Ferritin cut off $(36 \mathrm{ng} / \mathrm{ml})$ observed in $30 \%$ of FC cases without significant difference observed $(21 \%)$ of in non convulsive febrile controls ( $p$ value $=0.64)$. The ferritin as an acute phase reactant in all febrile patients might explain this no significant difference. This level has an acceptable sensitivity (74.3\%) but poor specificity (20\%) and just 56\% PPV, 52\% NPV to differentiate the FC cases from non-convulsive febrile children. 
The mean ferritin level (58) in all children in present study is very close to normal children (58.75),reported by Soheilipour et al (15), none of the children had ferritin level $<12 \mathrm{mic} /$.a significant difference between children with 3 ususal acute infections (GI, RTI, UTI) in compare with normal children $(60.05,62.00,60.60$, vs 58.75$)$ ).[15].

Association between ID and FC have shown contradictory results in Iran like the other countries. The higher rate for ID in Children with FC in some Iranian studies (14-18,22,24,25). In contrast, Bidabadi et al (19) reported that ID in FC was less than aferbrile controls (44\% vs $48 \%$ ) Salehi et al (21) observed that ID plays no role in pediatric FC. According to Fallah et all (22) ID was more frequent in FC cases ( $48 \%$ vs. $28 \%, p=0.04)$.

The power of present study is comparing the febrile children as controls not afebrile children. Lke us, Ghasemi et al reported ferritin level $(36 \mathrm{ng} / \mathrm{ml})$ in $40 \%$ of FC, $26 \%$ of children with non-convulsive febrile cases, $12 \%$ of healthy children (non-febrile, non-convulsive) (24). Sharif et al (25) reported ID in $45 \%$ of FC and $12 \%$ of non-convulsive febrile children (25) The $21 \%$ ID in non-convulsive febrile children (control group) in present study was just similar to $26 \%$ study by Hashemi et al (26\%). They realized that Children with FC were more likely to suffer from iron deficiency compared to those with solitary febrile illness or healthy children. Thus, ID could be considered as a risk factor for Fc, but in both mentioned studies, no cut-off point was determined for discrimination in three groups. ID could be an important risk factor for development of FC. Evaluation of iron status was encouraged to be performed in children with FC. Indeed, Sadeghzadeh et al (23) reported ID in $6 \%$ of FC cases in Zanjan; like as healthy groups. According to this study, it was suggested that although ID was not frequent in febrile seizure group of children, but iron deficiency was more reported in patients with Fever (23).

The peak incidence for FC is near 18 months age. (2-3) Height of temperature, positive family history of FC, maternal smoking and alcohol consumption during pregnancy, and perinatal exposure to antiretrovirals are probable risk factors for FC.(3) Although ,multiple etiologic factors are considered for FC such as molecular genetic basis(4), reduction of neurotransmitters ( zinc and magnesium „GABA) in pathogenesis of several seizure disorders $(5,6,7)$, low level of trace element in sera or CSF of children with FC (8-9) Changes in the levels of pro inflammatory cytokines,;prostaglandins and zinc in FC is reported (9)

In developing countries $46-66 \%$ of children under 4 years are anemic, with half attributed To ID (10) Pisacane et al.(11) showed the higher rate of ID in younger (<2yold) FC cases. Also, Kumar and Al Rahman showed lower level of ferritin in FC cases in compare with non-convulsive febrile children $(12,13)$. These previous results were totally contrary to results of both Iranian studies; $(22,24)$ and other studies carried out in other countries (11-13).

The cut off level for was not reached in none of previous studies in Iran, except for the present study. In our study, all patients that were chosen as patient group were admitted with their first FC, but in most previous mentioned studies, some Fc cases had a recent history of febrile convulsion. Various diagnostic criteria of ID in different references and ferritin level as an age dependent factor in diagnosis of ID might 
be another reason for unmatched results $(22,24,11)$. We are in favor of the results mentioned in Bidabadi et al (19) study that no identified relationship has been detected yet between ID and FC, maybe it could be considered as an accidental correlation or interference of other unknown factors such as genetic, viral and other transmitters (19). Talebian (20) reported the Pooled recurrent rate of febrile seizure in Iran was $20.9 \%$ (95\% Cl: $12.3-29.5)$. In $28.8 \%$ (95\% Cl: $19.3-38.4$ ) of children there was positive family history. They concluded that the risk of FC occurrence in children with ID seems to be more frequent than children with convulsive febrile seizure, ID could be considered as a protective factor against the risk of convulsion occurrence by raising the threshold of convulsion (20)

\section{Conclusion}

Inconsistency and conflict were detected in results of previous studies regarding the relationship between ID and FC In spite of this fact that fever could be considered as an aggravating factor in negative effects of iron deficiency on nervous system. In our opinion, ID could not lead to FC in all children, but in some cases with genetic basis; ID raises the threshold for seizures. Due to high prevalence of ID (26\%) especially in your Iranian population, adding the Iron to diet, might be helpful in decreasing FC in susceptible cases.

\section{Limitation Of Study}

The increasing of ferritin levels (above the bases line) as an acute phase reactant is acceptable in every febrile cases. We did not know the ferritin base level in each children (case/control) before infection.

\section{List Of Abbreviations}

Febrile convulsion (FC), Iron efficiency (ID), ELISA (enzyme-linked immunosorbent assay), ROC (receiveroperating-characteristic curve), PPV (positive predictive value); NPV (negative predictive value), Gamma Aminobutyric acid (GABA)

\section{Declarations}

\section{Ethics approval and consent to participate:}

This study was accredited by Ethical Committee of Iran University of Medical Sciences. Helsinki Declaration was respected across the study and the informed consent form was signed by the parents.

\section{Consent for publication:}

All authors confirm Consent for publication

\section{Availability of data and material:}

we confirm availability of data and materials in Records of hospital. 


\section{Competing interests:}

The authors declare no conflict of interest in preparing this study.

\section{Funding:}

This study received no specific grant from any funding agency in the public, institutions or not-for-profit sectors.

\section{Authors' contributions:}

Fahimeh Ehsanipour designed and supervised the study

Samileh Noorbakhsh:,corrosponder : writing the initial report and full article .

Azita Tavassoli: visited and interpreted the data.

Leila Tahernia: major contributors in rewriting and English editing the manuscript.

\section{Acknowledgment:}

We thanks all nursing staff in Pediarics department in Rasoul hospital affiliated by Iran university of medical sciences

\section{References}

1. Berg AT, Berkovic SF, Brodie MJ. Revised terminology and concepts for organization of seizures and epilepsies: Report of the ILAE Commission on Classification and Terminology, 2005-2009. Epilepsia 2010; 51: 676-685.

2. Fishman MA. Febrile seizures. (Web document) www.UptoDate.com Last Literature Review version; 8.13.

3. Østergaard J. R. Febrile Seizures. Acta Paediatr. 2009;98(5):771-3.

4. Nakayama, T. O. Arinami. Molecular genetics of febrile seizures. Epilepsy Res. 2006;70(1):190-8.

5. Loscher W, Rating D, Seiemes H. GABA in cerebrospinal fluid of children with febrile convulsions. Epilepsia. 1981;22(6):697-702.

6. Mollah MAH, Rakshit SC, Anwar KS, et al.Zinc concentration in serum and cerebrospinal fluid simultaneously decrease in children with febrile seizure Findings from a prospective study in Bangladesh. Acta Paediatr. 2008;97(12):1707-11.

7. Mishra OP, Singhal D, Upadhya RS, Prasad R, Atri D. Cerebrospinal fluid zinc, magnesium, copper and gammaaminobutyric acid levels in febrile seizure. J Pediatr Neurol. 2007;5:39-44.

8. Amiri, F, M, Sajadi. Serum trace element levels in febrile convulsion. Biol Trace Elem Res. 2010;135:38- 
9. Tutuncuoglu s, Kutukculer N, Kepe L, et al. Proinflammatory cytokines,prostaglandins and zinc in febrile convulsions. Pediatr Int. 2001;43(3):

10. R Masironi.The Epidemiology of Trace Element Deficiencies. Clinical Nutrition of the Essential Trace Elements and Minerals Nutrition $\diamond$ and $\diamond$ Health pp 91-97

11. Pisacane A, Sansone R, Impagliazzo N, Coppola A, Rolando P, D’Apuzzo A, et al. Iron deficiency anemia and febrile convulsion: Case control study in children under 2 years. BMJ, 1996; 313: 343.

12. Ur-Rehman N, Billoo A. G. Association between Iron Deficiency Anemia and Febrile Seizures. J Coll Physicians Surg Pak. 2005;15(6):338-40.

13. Kumar, S Bhushan, A Kumar.To Study the Association between Iron Deficiency Anemia and Febrile Convulsion in Children in a Tertiary Care Center- A Case Control Study. INDIAN JOURNAL OF APPLIED RESEARCHVol 7, No 6 (2017)

14. Akbari M, Moosazadeh M, Tabrizi R, Khatibi SR, Khodadost M, Heydari ST, Tahami AN, Lankarani KB Estimation of iron deficiency anemia in Iranian children and adolescents: a systematic review and meta-analysis. Hematology. 2017 May;22(4):231-239

15. Soheilipour F; Noorbakhsh S; Javadnia Sh Jesmi F; Pishgahroudsari M; Mohammadi Gh.Assessing the Serum Levels of Ferritin and Selenium in three Important Infections of Childhood, Compared to a Control Group .Current Nutrition \& Food Science:2015, 11(4): pp. 263-266

16. Mahyar A, Ayazi P, Fallahi M, Javadi A. Risk factors of the first febrile seizures in Iranian children. Int J Pediatr. 2010;2010:862897.

17. F Heydarian, *, E Bakhtiari , S Yousefi and MHeidarian. The First Febrile Seizure: An Updated Study for Clinical Risk Factors. Iran J Pediatr. 2018 December; 28(6):e69761.

18. Y Veisani; A Delpisheh; K Sayehmiri .Familial History and Recurrence of Febrile Seizures; a Systematic Review and Meta-Analysis . Int J Pediatr. 2010;2010:862-897.

19. Bidabadi E, Mashouf M. Association between iron deficiency anemia and first febrile convulsion: A case-control study. Seizure, 2009; 18(5): 347-51

20. Talebian A, Momtazmanesh N. Febrile Seizures and Anemia. Iran J Child Neurology. 2007:31-33.

21. Salehi Omran M. R, Tamaddoni A, Nasehi M. M, Babazadeh H, Alizadeh Navaei R. Iron Status in Febrile Seizure: A Case-control Study. Iran J Child Neurology. 2009:40-43.

22. R Fallah, MD, ${ }^{1}$ B Tirandazi, MD, ${ }^{2} S$ Akhavan Karbasi, $M D,{ }^{3}$ and $M$ Golestan, $M^{3}$ Iron Deficiency and Iron Deficiency Anemia in Children with Febrile Seizure. Iran J Ped Hematol Oncol. 2013; 3(1): 200203.

23. Sadeghzadeh M, Khoshnevis P, Mahboubi E. Iron Status and Febrile Seizure-A Case Control Study in Children Less Than 3 Years. Iran J Child Neurol. 2012;6(4):27-31.

24. F GHASEMI, F VALIZADEH, and N TAEE,Iron-deficiency Anemia in Children with Febrile Seizure: A Case-Control Study.Iran J Child Neurol. 2014 Spring; 8(2): 38-44.

25. MR Sharif, D Kheirkhah, M Madani, H H Kashani. The Relationship between Iron Deficiency and Febrile Convulsion: A Case-Control Study.Glob J Health Sci. 2016 Feb; 8(2): 185-189. 
Figures

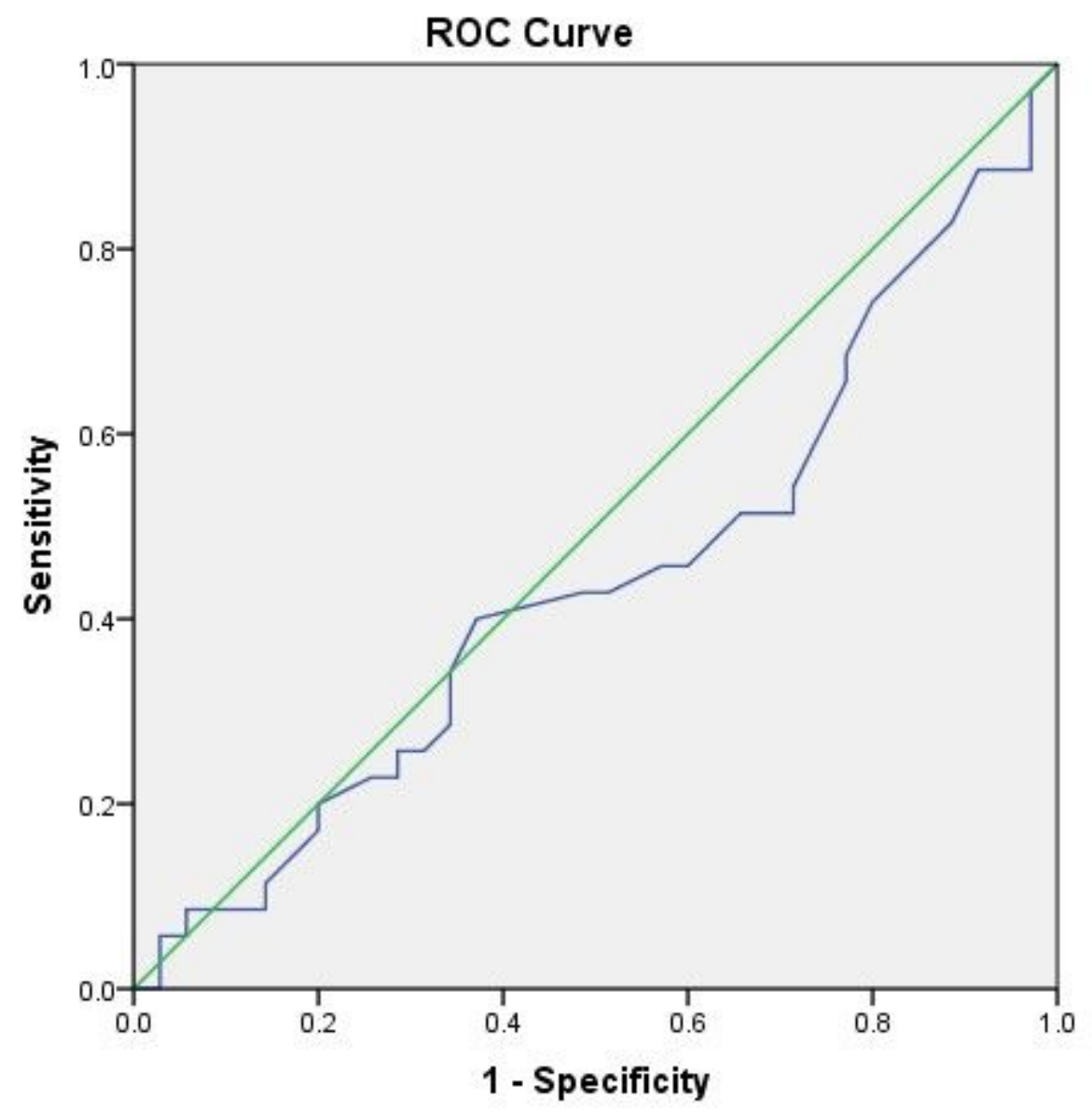

Diagonal segments are produced by ties.

\section{Figure 1}

Area under curve for Ferritin in ROC curve 